\title{
Outcomes with a posterior reduced dental arch: a randomised controlled trial
}

\author{
S. Khan, U. M. Chikte and R. Omar
}

\section{Summary}

To compare function, patient satisfaction and quality of life of patients with a posterior reduced mandibular arch with those who had all missing teeth replaced with removable partial dentures. Patients with at least three and not more than six posterior occluding pairs of teeth were enrolled sequentially and randomised into one of two treatment groups: a denture and no-denture group. A research assistant allocated interventions; concealment was ensured using opaque-sealed envelopes. Analysis of data was performed in stages, adding samples of 10 incrementally, and stopping when the relevant statistical tests indicated a clear conclusion as judged by the power set at $80 \%$ or above. Study outcomes included patient satisfaction, function and survival of remaining teeth at 3 and 12 months postintervention, using a visual analogue scale and the Oral Impacts on Daily Performance). Statistical analysis was performed by the 'intention-to-treat' principle. Age range of included patients was 2355 years $($ mean $=42 \cdot 3$; s.d. $=9 \cdot 2)$, with $78 \%$ being females. Most patients $(70 \%)$ belonged to the low- or no-income group. Nine patients left the study, for different reasons. Primary outcomes for the denture group: $10 \%$ of the patients were not satisfied and $20 \%$ were unhappy with their function; for the no-denture group: $85 \%$ of the patients (with $15 \%$ having left the study) were satisfied with both their function and their non-denture status. Patients with posterior reduced mandibular dental arches reported greater perceived satisfaction, function and quality of life compared to those who had received a cobalt- chrome clasp-retained partial removable prosthesis. KEYWORDS: prosthodontics, posterior reduced mandibular arch, randomised clinical trial, sequential sampling, patient satisfaction and function. 


\section{Introduction}

Research data increasingly support a functional approach in treatment planning. In prosthodontic clinical decision-making for older patients, such an approach not only encourages patient input, but has been shown to achieve improvements in subjective function and quality of life (QoL), thus ensuring overall treatment success (1-3). A functional approach also addresses the discrepancies that are known to exist between accepted normatively defi clinical practices and patients' evaluations of their oral functional needs $(1-6)$.

Results from several randomised and non-randomised clinical trials (RCT and CT) related to the shortened dental arch (SDA) concept have indicated its functional effectiveness, and application of the concept in selected patients has received general acceptance (4-11). Examination of these RCTs and CTs, however, highlights their differences, including the interventions used, aspects of study design and outcomes assessed (Table 1) (4-11). A recent systematic review on the SDA concluded that the results of the included studies were not always consistent, and that generalisability may only be possible for specific regional and, perhaps cultural contexts (12). As tooth loss and oral function are indicators of the oral health status of individuals and communities (13), their impact on the perceived need for replacement of missing teeth is critical $(2,14,15)$. Studies have indicated that the loss of teeth and their location significantly affect the oral health-related quality of life (OHRQoL) of patients $(2,6,15,16)$. The evidence for dentitions with fewer teeth, such as an extreme SDA confirms the negative effect on function and OHRQoL $(1,2,6,15)$. 


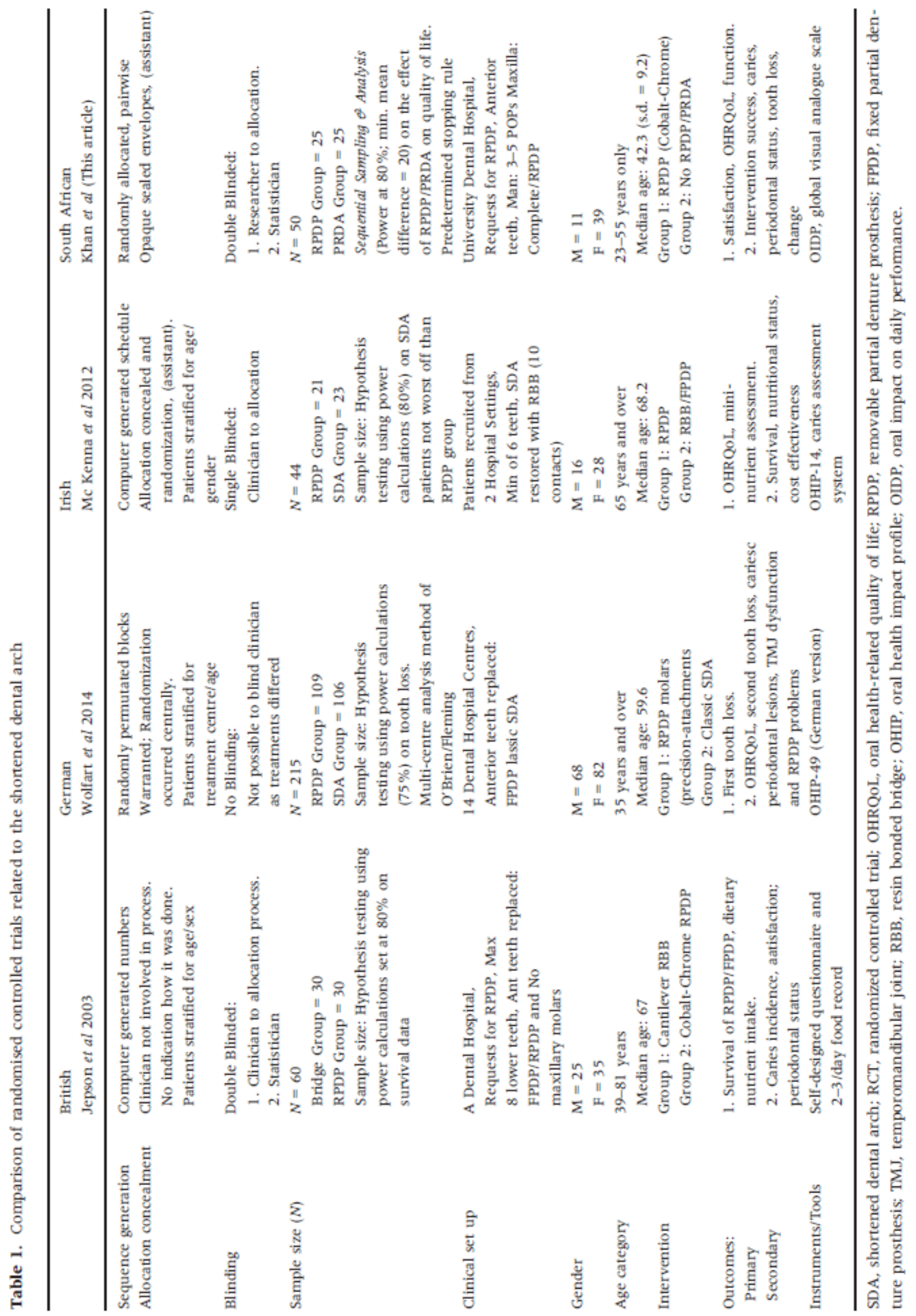


Of the several available instruments for measuring OHRQoL, the oral impact on daily performance (OIDP) tool is a multidimensional instrument that provides information related to oral conditions $(4-6,12,13,15-17)$. When used concurrently with clinical measures, a more comprehensive assessment of patients' oral status may be determined $(13,17)$. The OIDP has been validated, and together with a global visual analogue scale (VAS), may be used to assess oral status, patients' satisfaction and OHRQoL $(13,17)$.

Given the wide variations in missing posterior tooth distributions, the definition of a SDA has evolved $(2,3,15)$. A less formulaic, and perhaps more generic, clinical description may thus include a posteriorly reduced dental arch (PRDA) with 3-4 symmetrically- and 5-6 asymmetrically arranged posterior occluding pairs (POPs) of teeth $(1,2)$. In some situations, specific occlusal arrangements as in PRDAs which include the classic SDA are considered acceptable and adequate for oral function, occlusal support and stability $(2,15)$.

South Africa (SA) is a developing country, which by virtue of its wide socioeconomic disparities, affords only a limited range of treatment procedures for the majority of its population at public health clinics (viz. extractions, fillings and preventive procedures); at the same time, the exorbitant costs associated with current prosthodontic treatment options (complete or partial removable, or conventional or implant-retained fixed prostheses) that are provided by private practitioners make these options inaccessible for most. Management approaches such as the SDA or PRDA would seem to be an appropriate primary healthcare measure for the underprivileged majority of the population (18).

The aim of this study was to determine whether the daily functional needs and the quality of life of adult patients with a posterior reduced mandibular dental arch would be satisfied without having all their missing teeth replaced with a mandibular removable partial denture prosthesis (RPDP), as compared to having a prosthesis. The null hypothesis was that, in adult patients with a posterior reduced mandibular arch, there would be no difference in oral functional satisfaction and quality of life with or without the presence of a prosthesis to replace all missing teeth.

\section{Methods}

Ethical clearance was obtained from the Research and Ethics Committees of Stellenbosch University (Registration No: S13/04/o66) and University of the Western Cape (UWC) (Registration No: 12/5/14), SA. This single-centre doubleblinded RCT was designed according to the guidelines of the International Organization for Standardisation (ISO/EN540) and the Guidelines for Good Clinical Practice in SA (19, 20). Informed consent was obtained from all patients prior to commencement according to the Declaration of Helsinki (21). The results of this study are reported according to the Consolidated Standards of Reporting

\section{http://repository.uwc.ac.za}


Trials (CONSORT) statement $(19,22)$. The design aspects, study outcomes, data collection and follow-up details can be viewed in a detailed protocol and can be accessed at: clinicaltrials.gov; Identifier: NCT01597206.

Initially, the RCT sample recruited at the UWC dental hospital included patients with a classic SDA scheme for the mandible only, and requesting a RPDP. They were randomly allocated into one of two treatment approaches: Group A, with a cobalt-chrome RPDP as intervention; and Group B, with no RPDP (viz. a classic SDA), as control (19). In both groups, reduced and interrupted dentitions would first have been restored to the classic SDA scheme using fixed partial denture prostheses (FPDPs) (23).

The standard hypothesis testing method to estimate sample size, using the primary outcome of patient satisfaction, indicated that 420 patients (210 per study group) needed to be recruited. But after conducting a pilot study $(\mathrm{N}=6)$, patients with these specific clinical criteria were not easily obtainable. Thus, alternative recruitment criteria were set as follows: traditional sampling changed to sequential sampling; sourcing of patients was extended to include public health clinics; eligible mandibular arch types were modified from only classic SDAs to patients with three, and not more than six, POPs of teeth, and a complete natural maxillary arch or one rendered as complete by provision of either a complete or partial denture (2). For this double-blinded RCT, healthy young adult patients (21-55 years) having a mandibular PRDA with three and not more than six POPs formed the final sample (Table 1).

All basic restorative and preventive procedures were completed by the UWC servicerendering department, and the maxillary RPDP or complete denture and mandibular FPDPs were constructed by a clinical assistant according to standard clinical protocols (23). Patients were randomly entered and interventions allocated by a research assistant using sealed opaque envelopes into: Group A to replace all missing mandibular posterior teeth with a cobalt-chrome clasp-retained RPDP following standard prosthodontic design principles and constructed by the clinical assistant; or Group B with a mandibular PRDA $(17,23)$ (Fig. 1).

The following subjective and objective outcomes with the mandibular intervention were determined:

Primary outcomes: patient satisfaction, oral function and OHRQoL; and Secondary outcomes: clinical performance, survival of remaining teeth and mandibular RPDP (caries, periodontal problems, loss of teeth or inability to wear the RPDP), or a change in treatment allocated.

Evaluation of the outcomes was performed by the principal researcher 3 and 12 months after receiving the intervention, as applicable, using the global VAS and OIDP $(13,17,24)$. The global VAS is a $100 \mathrm{~mm}$ scale comprising five questions which 
focused on patient satisfaction, need for treatment and quality of life regarding the current state of their teeth and the intervention provided.

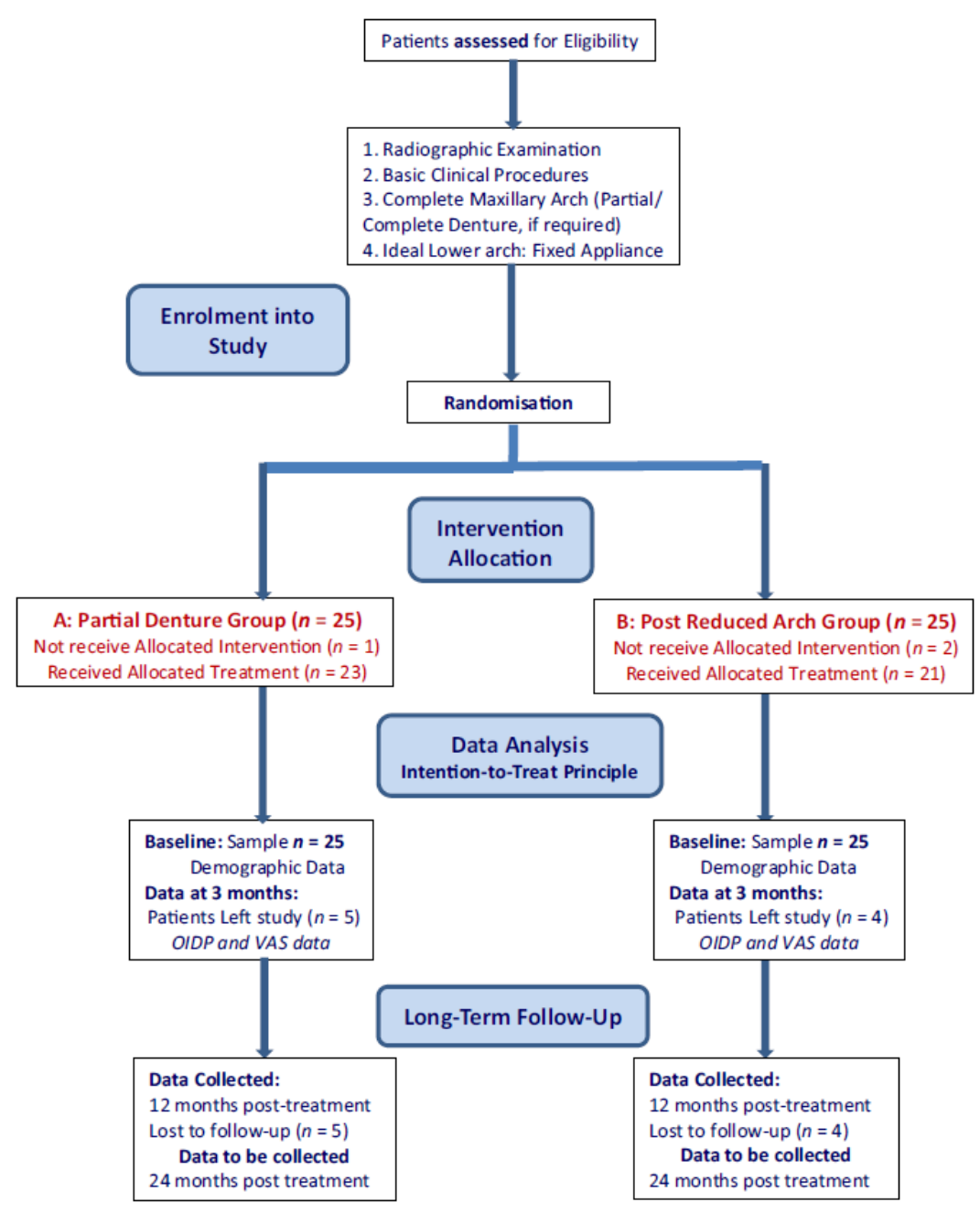

Fig. 1. Patient flow diagram.

Questions at baseline and prior to provision of the intervention, and questions 4-5 were completed 3 months after receiving the intervention (17). The specific oral impacts questions in the OIDP relating to OHRQoL measures include oral function, oro-facial appearance and psychological impact (13). The OIDP gave an overall rating of patients' satisfaction as well as oral health, QoL and OHRQoL.

Statistical analysis of data was completed by the 'intention-to-treat' principle, and patients' personal details were omitted for this phase (25). Analysis included finalising the sample size, frequency calculations of demographic data, oral impacts and VAS scores, calculation of correlation coefficient and comparisons using the Chi-square test (25). It also included primary outcomes investigation and adjustment for confounding, where necessary. 


\section{Results \\ Sampling for the study}

Sampling was by necessity sequential, and the data were similarly analysed sequentially. Because VAS questions 4 and 5 were related to the intervention (i.e. 'the impact of the intervention on the patients' oral health' and 'quality of life', respectively), they were used as the primary variables upon which the conclusion to stop sampling was based $(26,27)$ (Table 2). Patients were included as they presented for treatment and the allocation of mandibular intervention was made pairwise into the two study groups A and B. Sample size was not fixed in advance but finalised as data was obtained. For this purpose, a pre-defined stopping rule had to be set:

1. If the estimated power was greater than $80 \%$, accept either the null or alternative hypothesis and stop sampling, or

2. Continue sampling and increase the sample size incrementally by 10 patients $(26,27)$.

Assessment of data collected was performed sequentially on sets of $N=10$ patients, using a two-sample $t$-Test to determine the power of the study which was set at $80 \%$ and above. The first set of $N=10$ patients was thus Stage 1 of the sequential process, and $N=20$ was Stage 2, and so on. For this assessment, a mean difference of 20 (which was a figure considered by the researchers to indicate the smallest difference that may be considered clinically important) between the two groups for variables VAS4 and VAS5 and a statistical significance of $0 \cdot 05$ was set (25). The decision to continue sampling was based on the power determined at each $N=10$ increment; further sampling and analysis, which would similarly be completed sequentially, stopped as significant results were obtained $(26,27)$.

At Stage $1(N=10)$ and Stage $2(N=20)$, the power determined was below $80 \%$ and thus unacceptable; recruitment of further sets of patients thus continued (Table 2). At Stage 3, the sample size was acceptable $(N=30)$ on the basis that the power of the study was calculated as $80 \%$ and above $(26,27)$ (Table 2). At this stage, further recruitment of patients could have been stopped, but we wanted to see the effects on outcomes with additional groups of 10 participants $(N=40$ and $N=$ 50) (Table 2). 
Table 2. Sequential sampling calculations using a two-sample $t$-test

\begin{tabular}{|c|c|c|c|c|c|c|}
\hline $\begin{array}{l}\text { Sample size } \\
(N) \text { per stage }\end{array}$ & $\begin{array}{l}\text { VAS } \\
\text { question }\end{array}$ & $\begin{array}{l}\text { Sample size } \\
\text { for analysis }\end{array}$ & $\begin{array}{l}\text { Minimum } \\
\text { mean difference }\end{array}$ & $\begin{array}{l}\text { Standard } \\
\text { deviation, s.d. }\end{array}$ & $\begin{array}{l}\text { Statistical } \\
\text { significance }\end{array}$ & Power \% \\
\hline \multirow[t]{2}{*}{$N=10$} & 4 & 5 & $20^{\star}$ & 20.28 & 0.05 & $27.9 \%$ \\
\hline & 5 & 5 & 20 & 22.86 & 0.05 & $23 \%$ \\
\hline \multirow[t]{2}{*}{$N=20$} & 4 & 10 & 20 & 15.40 & 0.05 & $78.4 \%$ \\
\hline & 5 & 10 & 20 & 17.11 & 0.05 & $69.5 \%$ \\
\hline \multirow[t]{2}{*}{$N=30$} & 4 & 13 & 20 & 13.92 & 0.05 & $93.9 \%$ \\
\hline & 5 & 13 & 20 & 16.43 & 0.05 & $84 \%$ \\
\hline \multirow[t]{2}{*}{$N=40$} & 4 & 17 & 20 & 12.39 & 0.05 & $99.4 \%$ \\
\hline & 5 & 17 & 20 & 14.61 & 0.05 & $97.4 \%$ \\
\hline \multirow[t]{2}{*}{$N=50$} & 4 & 20 & 20 & 18.36 & 0.05 & $91.9 \%^{\dagger}$ \\
\hline & 5 & 20 & 20 & 19.15 & 0.05 & $90.2 \%^{\dagger}$ \\
\hline
\end{tabular}

VAS, Visual Analogue Scale (100 mm ruler); VAS Question 4, How would you rate the effect of the intervention on your mouth/oral health? (Responses: Very Bad to Excellent); VAS Question 5, How would you rate the effect of the intervention on your quality of life? (Responses: Very Bad to Excellent).

${ }^{*}$ Minimum Mean difference for VAS4 and VAS5 which are considered clinically important and are required when determining the Power of the $t$-test

${ }^{\dagger}$ The power calculated decreased as the data induded an unexpected extreme response (an OUTLIER)

\section{Demographic data obtained at baseline}

Fifty patients were included in the RCT, with ages ranging from 23 to 55 years (mean $=42 \cdot 3$; s.d. $=9 \cdot 2)$, and with a bias towards the female gender at $39(78 \%)$ (Table 3). Education level of patients indicated that $41(82 \%)$ had been to school. Many worked in the public sector, 19 (38\%) in all, or were unemployed, 26 (52\%). Seventy per cent were in the 'low' or 'no-income' category. The periodontal status of the group at baseline was acceptable (a requirement to be enrolled into the study) with acceptable oral hygiene practices, with $38(76 \cdot 47 \%)$ brushing teeth twice a day).

\section{Patient satisfaction, QoL and OHRQoL}

At baseline, using the global VAS (0-100 mm scale): 41 (84\%) had a score of below $50 \mathrm{~mm}$ and rated the state of their mouth or teeth poorly, while 42 (84\%), with a score of $50 \mathrm{~mm}$ or less, were not satisfied with their current oral status. Forty-nine (92\%) of included patients, with scores ranging from 50 to $100 \mathrm{~mm}$, felt that they were in need of treatment (Table 3).

Three months after receiving the intervention (mandibular denture or no denture), with reference to the OIDP questions 8-10: 40 (80\%) of all patients indicated an acceptable dental health, and 36 (76\%) an acceptable patient satisfaction rating (Table 3). The OIDP questions were completed after all basic restorative or preventive procedures were completed. Three months after receiving the mandibular intervention, only participants in the 'denture group' rated the effect of the RPDP on their oral health and quality of life negatively, relating to questions 4 and 5 on the global VAS (Table 3). 


\section{Correlation between $\mathrm{VAS}$ and OIDP results}

For satisfaction, the VAS1 question ( $84 \%$ not satisfied with their oral state) was completed prior to treatment, while the related OIDP question $(76 \%$ satisfied and very satisfied with their oral state) was completed 3 months postmandibular intervention (Fig. 2, Table 4). As the VAS4 score (50 $\mathrm{mm}$ and above) for 'rating the effect of the intervention on oral health' increased, patient satisfaction also increased $(P=0 \cdot 05)$. Similarly, 'rating the effect of the intervention on quality of life' increased (as reflected in VAS5 scores of $50 \mathrm{~mm}$ or more), thus increasing patient satisfaction $(P=0.05)$. Both VAS4 and VAS5 scores (i.e. 'the impact of the intervention on the patients' oral health' and 'quality of life', respectively) indicated a negative correlation (viz. a decrease) with the need for treatment (Table 4).

\section{Oral impacts}

Oral impacts for measures relating to oral function, orofacial appearance and psychological impact, and an overall health rating were fully explored to the extent that OIDP permitted, but only significant results are reported. Total OIDP score measures prevalence (proportion of subjects reporting one or more oral impact), extent (number of daily performances impacted) and severity (more severe effect in one performance) of oral impacts on daily life using a 5-point Likert scale (13). 
Table 3. Detailed comparison between two intervention groups

\begin{tabular}{|c|c|c|}
\hline & $\begin{array}{l}\text { Posterior reduced } \\
\text { dental arch group }\end{array}$ & $\begin{array}{l}\text { Denture } \\
\text { group }\end{array}$ \\
\hline \multicolumn{3}{|l|}{ Pre-Intervention } \\
\hline \multicolumn{3}{|l|}{ Baseline data } \\
\hline Sample $(N)$ recruited & 25 & 25 \\
\hline Gender (Females) & 21 & 18 \\
\hline Full Maxillary Denture & 5 & 7 \\
\hline VAS $1(0-50 \mathrm{~mm})$ & 22 & 19 \\
\hline VAS $2(0-50 \mathrm{~mm})$ & 21 & 21 \\
\hline VAS $3(50-100 \mathrm{~mm})$ & 25 & 24 \\
\hline \multicolumn{3}{|l|}{ Post-Intervention } \\
\hline \multicolumn{3}{|l|}{3 Months } \\
\hline VAS $4(65-100 \mathrm{~mm})$ & 21 & 18 \\
\hline VAS $5(58-100 \mathrm{~mm})$ & 21 & 18 \\
\hline OIDP 8: Good & 21 & 19 \\
\hline OIDP 9: Satisfied & 19 & 17 \\
\hline OIDP 10: No treatment & 20 & 18 \\
\hline OIDP: 13a (eating) & 1 & 4 \\
\hline OIDP: 13b (speaking) & 0 & 2 \\
\hline OIDP: 13i (emotional) & 1 & 2 \\
\hline \multicolumn{3}{|l|}{ Primary outcomes } \\
\hline Patient satisfaction & 21 & 18 \\
\hline Function & 21 & 14 \\
\hline \multicolumn{3}{|l|}{ Secondary outcomes } \\
\hline Success of treatment & 21 & 15 \\
\hline Treatment change & 2 & 2 \\
\hline Patient loss & 4 & 5 \\
\hline
\end{tabular}

VAS, visual analogue scale; OIDP, oral impact of daily performance

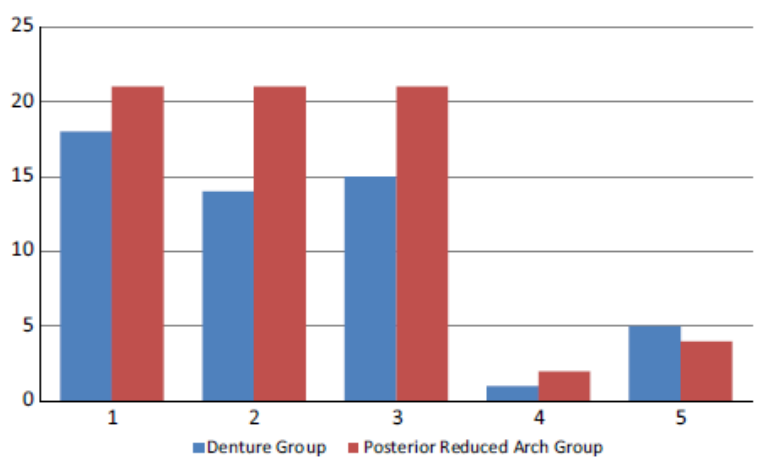

Fig. 2. Primary and secondary outcomes reported with the interventions in the mandibular arch: 'denture' or 'posterior reduced dental arch' (viz. 'no denture'). Primary outcomes: 1. Patient satisfaction with intervention; 2. Function with intervention. Secondary outcomes: 3 . intervention is a success; 4. Treatment changed; 5 . Number of patients left study. 
Table 4. Correlation coefficients for VAS questions versus OIDP questions rating oral health, patient satisfaction and need for dental treatment

\begin{tabular}{lrlr}
\hline & \multicolumn{2}{c}{ Oral Impacts on daily performance questions } \\
\cline { 2 - 4 } & $\begin{array}{l}\text { Dental } \\
\text { health }\end{array}$ & $\begin{array}{l}\text { Patient } \\
\text { satisfaction }\end{array}$ & $\begin{array}{l}\text { Need for } \\
\text { treatment }\end{array}$ \\
\hline VAS Questions & -0.003 & 0.109 & -0.261 \\
State of mouth & -0.018 & 0.014 & -0.256 \\
Satisfaction & -0.042 & -0.130 & 0.225 \\
Need treatment & -0.566 & $0.628(P=0.05)^{*}$ & $-0.536^{*}$ \\
Intervention & & & \\
$\quad$ on mouth & & $0.648(P=0.05)^{*}$ & -0.452 \\
Intervention & 0.465 & & \\
on quality of life & & & \\
\hline
\end{tabular}

VAS, visual analogue scale; OIDP, oral impacts on daily performance.

PRE-INTERVENTION (Baseline): Questions VAS1, VAS2 and VAS 3; POST-INTERVENTION (3 months Post Intervention): Questions VAS4, VAS5, OIDP8, OIDP9 and OIDP10.

*Indicate significant correlations

Total OIDP score (2.98\%) recorded after receiving the mandibular intervention was very low, signifying a good self-rated health status. Only one patient reported all oral impacts as negative, with six patients having problems with the oral impacts of eating, and three having negative feelings of being emotional. These were experienced daily for the one patient, and once a month for the others with similar effects on their daily life.

\section{Outcomes reporting}

From a sample of 50, nine patients left the study: four from the 'no-denture' and five from the 'denture' group (Fig. 2). Reasons for leaving included the following: unhappy with being allocated to the 'no-denture' group, losing teeth, moving cities and work commitments. Only two of these patients continued with a change in treatment (Fig. 1).

Data related to the primary outcomes obtained 3 months after receiving the mandibular intervention indicated that, for the 'denture group', $4 \%$ were not satisfied, $12 \%$ were unhappy with their function, each of which negatively affected the success with the allocated intervention (Fig. 2). In comparison, for the 'no-denture group', all of those who remained in the study were satisfied with their non-denture status and content with their function.

Regarding clinical performance, two patients complained about adapting to the mandibular RPDP and another mentioned the instability of the lower free-end saddle. No other negative secondary outcomes were reported by either group at this stage (Fig. 2). 
One year after treatment, no negative reports were received regarding patients' PRDA status or any other secondary outcomes. However, reports of adaptation to RPDPs (both upper and lower), the need for a restoration in the maxillary arch and the usual checkups were recorded.

\section{Discussion}

Our main finding in this RCT was that patients with a PRDA on the mandible reported greater satisfaction, and perceived success of treatment relating to function and OHRQoL without a RPDP compared to those who had had their missing teeth replaced with a cobalt-chrome clasp-retained mandibular RPDP. This was encouraging given the known constraints on access to conventional prosthodontic treatment for a large proportion of partially dentate patients, especially in developing countries. A functional approach to treatment planning that the present findings would appear to support also addresses the differences that are known to exist between normatively defined clinical practices and patients' evaluations of their oral functional needs (1-6). Furthermore, none of the present PRDA patients not provided with a RPDP expressed the need to have their missing mandibular teeth replaced 12 months post-treatment. Clinically, the significance of these results cannot be overstated especially coming from a resource-constrained setting such as SA.

A not infrequent concern of patients allocated to the 'denture group' was regarding the use of distal extension mandibular dentures, which has also been reported in the literature $(1-4,14,28,29)$. These concerns typically relate to 'adapting to dentures' and the 'high expectations' patients have with RPDPs (3, 10, 23, 28, 29). Equally, the positive responses from the 'no-denture group' that imply acceptable function, satisfaction and OHRQoL with a PRDA concur with extensive literature elsewhere, albeit whose context was not identical with the present study (1-10, $15,16,18,29,30)$.

The sequential sampling used in the present study made it possible to purposefully limit the sample size. Thus, patients' responses were statistically validated when the analysis indicated no difference in their responses, from one staged point to the next, when comparing denture-wearing to non-denturewearing patients as regards function, comfort, aesthetics, patient satisfaction and OHRQoL. Moreover, several primary and secondary research studies have concluded that the SDA treatment option is justified on the basis of reduced costs, patient satisfaction and temporomandibular concerns (1-10, 15, 16, 18, 29, 30). Lastly, problems experienced by patients with mandibular RPDP usage were comparable with those previously reported as it relates to function, comfort, aesthetics, limitations of denture-wearing, increase in root caries formation and costs of RPDPs $(1-12,14-16,18,28-30)$. 
The clinical implications of these results emphasise the need for evidence-based practices. Patients are receptive to such alternative treatments, especially when the clinician has adequately educated and guided them to practices that would be beneficial to them. Approaches such as the SDA or PRDA may be considered primary healthcare measures and may address the widespread socio-economic constraints.

A RCT study design is by its very nature challenging. Making changes to what is already a complicated design may present with even more difficulties. The sampling method adopted in this RCT is fairly novel and has rarely been used in clinical dental research, so that its implementation may be regarded as a limitation. While a small sample size may be construed as a limitation, an explanation following statistical validation has been provided. Nevertheless, some researchers may disagree about the generalisability of the results to the population at large given the small sample size. Gender bias may also be considered a limitation, but the random inclusion of patients was from the general population who were in need of denture treatment and who visited the University and general public hospitals. No stratification for age or medical conditions was conducted and this may also be regarded as a limitation. Moreover, the exclusion of patients treated with FPDPs or implant-retained prostheses, and the use of one examiner for recalls may also be considered as limitations.

\section{Conclusion}

Patients with a mandibular PRDA reported greater satisfaction, perceived success of treatment relating to function and OHRQoL without a RPDP compared to those with a complete dental arch that was extended with a cobalt-chrome claspretained RPDP.

\section{Acknowledgments}

The study was funded by the University of the Western Cape and Stellenbosch University, South Africa. The authors also thank everyone who participated in this clinical trial.

\section{Conflict of Interest}

All authors declare that they have no conflict of interest with regard to the content of the manuscript or funding of the study. 


\section{References}

1. Kayser AF. The shortened dental arch: a therapeutic concept in reduced dentitions and certain high risk groups. Int J Perio Rest Dent. 1989;9:426-449.

2. Gotfredsen K, Walls AWG. What dentition assures oral function? Clin Oral Impl Res. 2007;18:34 .

3. Fejerskov O, Escobar G, JӨssing M, Baelum V. A functional natural dentition for all - and for life? The oral healthcare system needs revision. J Oral Rehabil. 2013;40:707-722.

4. Jepson NJA, Allen PF, Moynihan PJ, Kelly PJ, Watson GW, Thomason JM. Patient satisfaction following restoration of shortened mandibular dental arches in a randomized controlled trial. Int J Prosthodont. 2003;16:409-414.

5. Wolfart S, Muller F, Gerß J, Heydecke G, Marre B, B£ning K et al. The randomized shortened dental arch study: oral-health-related quality of life. Clin Oral Invest. 2014;18:525- 533 .

6. Gerritsen AE, Allen FP, Witter DJ, Bronkhorst EM, Creugers NHJ. Tooth loss and oral health-related quality of life: a systematic review and meta-analysis. Health Qual. Life Outcomes. 2010;8:126-139.

7. Walter MH, Hannak W, Kern M, Mundt T, Gernet W, Weber A et al. The randomized shortened dental arch study: tooth loss over five years. Clin Oral Invest. 2012;17:877-886.

8. McKenna G, Allen PF, Flynn A, O’Mahony D, DaMata C, Cronin M et al. Impact of tooth replacement strategies on the nutritional status of partiallydentate elders. Gerodontology. 2012;29:e883-e890.

9. McKenna G, Allen PF, Woods N, O’Mahony D, DaMata C, Cronin M et al. A preliminary report of the cost-effectiveness of tooth replacement strategies for partially-dentate elders. Gerodontology. 2012;30:207-213.

10. Budtz-Jœrgensen E, Isidor F. 5-year longitudinal study of cantilever fixed partial dentures compared with a removable partial denture in a geriatric population. J Prosthet Dent. 1990;64:42-47.

11. Isidor F, Budtz-Jœrgensen E. Periodontal conditions following treatment with a distally extending cantilever bridge with removable partial dentures in elderly patients: a 5-year study. J Periodontol. 1990;61:21-26.

12. Khan S, Musekiwa A, Chikte UME, Omar R. Differences in functional outcomes for adult patients with prosthodontically-treated and -untreated Shortened Dental Arches: a systematic review. PLoS One. 2014;9:e101143.

13. Hobdell M, Tsakos G, Ladrillo TE, Ross MW, Myburgh N, Gordon N et al. Using an oral health-related quality of life measure in three cultural settings. Int Dent $\mathrm{J}$. 2009;59:381-388.

14. Cruegers NHJ, Witter DJ, Van"t Spijker A, Gerritsen AE, Kreulen CM. Occlusion and Temporomandibular function among subjects with mandibular distal extension removable partial dentures. Int J Dent. 2010;807850:1-7 https://doi. org/10.1155/2010/807850.

15. Elias EC, Sheiham A. The relationship between satisfaction with mouth and number and position of teeth. J Oral Rehabil. 1998;25:649-661.

\section{http://repository.uwc.ac.za}


16. Baba K, Igarashi Y, Nishiyama A, John MT, Akagawa Y, Ikebe K et al. The relationship between missing occlusal units and oral health-related quality of life in patients with shortened dental arches. Int J Prosthodont. 2008;21:72-74.

17. Lamb DJ, Ellis B. Comparison of patient self-assessment of complete mandibular denture security. Int J Prosthodont. 1996;9:309-314.

18. Owen CP. Appropriatech: prosthodontics for the many, not just for the few. Int J Prosthodont. 2004;17:261-262.

19. Webb P, Bain C, Pirozzo S. Essential epidemiology: an introduction for students and health professionals. Cambridge, UK; New York: Cambridge University Press; 2005 .

20. International Organisation for Standardisation. European Standard (ISO/EN 540). Berlin, Germany: Clinical Investigation of Medical Devices for Human Subjects; 2011.

21. World Medical Organization. Declaration of Helsinki. Br Med J. 1996;313:14481449.

22. Moher D, Schulz KF, Altman DG. CONSORT Group. The CONSORT statement: revised recommendations for improving the quality of reports of parallel-group randomised trials. Lancet. 2001;357:1191-1194.

23. Zarb GA, Bolender CL, Carlsson GE, Boucher CO. Boucher's prosthodontic treatment for edentulous patient. London: McGraw-Hill; 2004.

24. Sutton AF, Worthington HV, Mc Cord JF. RCT comparing posterior occlusal forms for complete dentures. J Dent Res. 2007;86:651-655.

25. Dawson B, Trapp RG. Basic and clinical biostatistics, 4th edn. New York, NY: Lange Medical Books/McGraw-Hill Publishing Division; 2004.

26. Armitage $\mathrm{P}$. Sequential methods in clinical trials. Am J Public Health. 1958;48:1995-2002.

27. Donaldson AN, Whitehead J, Stephens R, Machin D. A simulated sequential analysis based on data from two MRC trials. Br J Cancer. 1993;68:1171-1178. 28. Frank RP, Brudvik JS, Le Roux B, Milgrom P, Hawkins N. Relationship between the standards of removable partial denture construction, clinical acceptability and patient satisfaction. J Prosthet Dent. 2008;83:521-527.

29. Witter DJ, van Elteren P, Kayser AF, Van Rossum MJM. The effect of removable partial dentures on oral function in shortened dental arches. J Oral Rehabil. 1989;16:27-33.

30. Sarita PT, Witter DJ, Kreulen CM, Van't Hof MA, Creugers NHJ. Chewing ability of subjects with shortened dental arches. Community Dent Oral Epidimiol. 2003;31:328. 